PROCEEDINGS OF THE

AMERICAN MATHEMATICAL SOCIETY

Volume 126, Number 7, July 1998, Pages 2047-2052

S $0002-9939(98) 04326-3$

\title{
A REMARK ON NORMAL DERIVATIONS
}

\author{
B. P. DUGGAL
}

(Communicated by Palle E. T. Jorgensen)

\begin{abstract}
Given a Hilbert space $H$, let $A, S$ be operators on $H$. Anderson has proved that if $A$ is normal and $A S=S A$, then $\|A X-X A+S\| \geq\|S\|$ for all operators $X$. Using this inequality, Du Hong-Ke has recently shown that if (instead) $A S A=S$, then $\|A X A-X+S\| \geq\|A\|^{-2}\|S\|$ for all operators $X$. In this note we improve the Du Hong-Ke inequality to $\|A X A-X+S\| \geq\|S\|$ for all operators $X$. Indeed, we prove the equivalence of Du Hong-Ke and Anderson inequalities, and show that the Du Hong-Ke inequality holds for unitarily invariant norms.
\end{abstract}

\section{INTRODUCTION}

Let $H$ be a complex infinite dimensional Hilbert space, and let $B(H)$ denote the algebra of operators (i.e., bounded linear transformations) on $H$. Given $A, B \in$ $B(H)$, define the generalised derivation $C(A, B): B(H) \rightarrow B(H)$ by $C(A, B)(X)=$ $A X-X B$, and let $R(A, B): B(H) \rightarrow B(H)$ be defined by $R(A, B)(X)=A X B-X$. Taking $A=B$ to be a normal operator, J. Anderson [1] has shown that if $S$ is an operator in the commutator of $A$ (i.e., $C(A, A)(S)=0)$, then

$$
\|C(A, A)(X)+S\| \geq\|S\|
$$

for all $X \in B(H)$. Inequality (1) has an $R(A, A)$ analogue. Thus if $A$ is a normal operator such that $R(A, A)(S)=0$ for some $S \in B(H)$, then

$$
\|R(A, A)(X)+S\| \geq\|A\|^{-2}\|S\|
$$

for all $X \in B(H)$, and if $A$ is a contraction such that $R\left(A, A^{*}\right)(S)=0=$ $R\left(A^{*}, A\right)(S)$ for some $S \in B(H)$, then

$$
\left\|R\left(A, A^{*}\right)(X)+S\right\| \geq\|S\|
$$

for all $X \in B(H)$ (see [2, Theorems 1 and 2]). As stated in [1], inequality (1) says that the range of $C(A, A)$ is in a certain sense orthogonal to the kernel of $C(A, A)$. A similar remark applies to inequalities (2) and (3). The orthogonality (in the usual sense) of the range and the kernel of normal derivations $C(A, A)$ with respect to the (wider) class of unitarily invariant norms on $B(H)$ has been considered by Fuad Kittaneh [5].

Received by the editors January 31, 1996 and, in revised form, December 18, 1996.

1991 Mathematics Subject Classification. Primary 47A30, 47A63, 47B15, 47B48.

Key words and phrases. Normal derivation, norm inequality, contraction, unitarily invariant norm.

(C)1998 American Mathematical Society 
Notice that if we let $\widehat{A}=A \oplus A^{*}, \widehat{X}=\left[\begin{array}{ll}0 & X \\ 0 & 0\end{array}\right]$ and $\widehat{S}=\left[\begin{array}{ll}0 & S \\ 0 & 0\end{array}\right]$ on $\widehat{H}=H \oplus H$, then

$$
\|R(A, A)(X)+S\|=\left\|R\left(\widehat{A}, \widehat{A}^{*}\right)(\widehat{X})+\widehat{S}\right\| .
$$

We consider $R\left(A, A^{*}\right)$, and show that the constant $\|A\|^{-2}$ in inequality (2) may be replaced by 1 . The proof of inequality (2) in [2] makes essential use of Anderson's inequality: we prove the equivalence of inequalities (1) and (2) (by independently proving (2) and then deducing (1)). Given a normal operator $A, R\left(A, A^{*}\right)(X)=0$ implies $R\left(A^{*}, A\right)(X)=0$. Let us call a pair of operators $\left(A, B^{*}\right)$ a PF-pair (short for Putnam-Fuglede pair) if $R(A, B)(X)=0$ implies $R\left(A^{*}, B^{*}\right)(X)=0, X \in B(H)$. We extend inequality (2) to certain non-normal PF-pairs $\left(A, B^{*}\right)$, and (also) to the class of unitarily invariant norms on $B(H)$. The hypothesis $R\left(A, A^{*}\right)(S)=0=$ $R\left(A^{*}, A\right)(S), A$ a contraction, implies that the restriction of $A$ to the closure of the range of $S$ is unitary; we use this observation to deduce inequality (3) from our inequality (2).

\section{REsults}

In addition to the notation already introduced, we shall use the following further notation. Given $X \in B(H)$, we shall denote the kernel, the orthogonal complement of the kernel and the closure of the range of $X$ by $\operatorname{ker} X, \operatorname{ker}^{\perp} X$ and $\overline{\operatorname{ran} X}$, respectively. The spectrum, the approximate point spectrum and the point spectrum of $X$ will be denoted by $\sigma(X), \sigma_{\pi}(X)$ and $\sigma_{p}(X)$, and the restriction of $X$ to an invariant subspace $M$ will be denoted by $X \mid M$.

Given $A \in B(H)$, there exists a Hilbert space $H^{\circ} \supset H$ and an isometric *isomorphism $A \rightarrow A^{\circ}$ such that $\sigma(A)=\sigma\left(A^{\circ}\right)$ and $\sigma_{\pi}(A)=\sigma_{\pi}\left(A^{\circ}\right)=\sigma_{p}\left(A^{\circ}\right)$. This is the Berberian extension theorem [6, p. 15].

The following theorem is the main result of this paper.

Theorem 1. If $A$ is a normal operator such that $R\left(A, A^{*}\right)(S)=0$ for some $S \in$ $B(H)$, then

$$
\left\|R\left(A, A^{*}\right)(X)+S\right\| \geq\|S\|
$$

for all $X \in B(H)$.

Proof. Use the Berberian extension theorem to obtain a Hilbert space $H^{\circ}$ and operators $A^{\circ}, S^{\circ}$ (on $H^{\circ}$ ) such that $R\left(A^{\circ}, A^{\circ *}\right)\left(S^{\circ}\right)=0$. The operator $A$ being normal, $A^{\circ}$ is normal, and hence $R\left(A^{\circ *}, A^{\circ}\right)\left(S^{\circ}\right)=0$ (see [3]). Consequently, $\overline{\operatorname{ran} S^{\circ}}$ reduces $A^{\circ}$ and $\operatorname{ker}^{\perp} S^{\circ}$ reduces $A^{\circ *}$. Let $A^{\circ}=B \oplus B_{\circ}$ with respect to $H^{\circ}=\overline{\operatorname{ran} S^{\circ}} \oplus\left(\overline{\operatorname{ran} S^{\circ}}\right)^{\perp}$, and let $A^{\circ *}=C^{*} \oplus C_{\circ}^{*}$ with respect to $H^{\circ}=\operatorname{ker}^{\perp} S^{\circ} \oplus$ $\operatorname{ker} S^{\circ}$. Define the quasi-affinity $Y: \operatorname{ker}^{\perp} S^{\circ} \rightarrow \overline{\operatorname{ran}} S^{\circ}$ by setting $Y x=S^{\circ} x$ for each $x \in \operatorname{ker}^{\perp} S^{\circ}$. Then $R\left(B, C^{\star}\right)(Y)=0$, where $B, C$ are normal, $\sigma(B)=\sigma_{p}(B)$ and $\sigma(C)=\sigma_{p}(C)$. Since $\operatorname{ker} B=\operatorname{ker} B^{*}, R\left(B, C^{*}\right)(Y)=0$ implies that if $x \in \operatorname{ker} B$, then $x \in \operatorname{ker} Y^{*}$. Hence, since $Y$ is a quasi-affinity, $0 \notin \sigma_{p}(B)$. Similarly, $0 \notin \sigma_{p}(C)$. Also, since $R\left(B, C^{\star}\right)(Y)=0$ implies $R\left(B^{*}, C\right)(Y)=0$ (by the normality of $B$ and $C), C^{*-1}$ is unitarily equivalent to $B$, i.e., there exists a unitary $U: \overline{\operatorname{ran} S^{\circ}} \rightarrow$ $\operatorname{ker}^{\perp} S^{\circ}$ such that $C^{*}=U B^{-1} U^{*}$.

Let $\lambda_{1}, \lambda_{2}, \ldots$ be an enumeration of distinct eigen-values of $B$, and let $M_{i}=$ $\operatorname{ker}\left(B-\lambda_{i}\right)$. Since $\sigma\left(C^{*}\right)=\sigma\left(B^{-1}\right), \sigma\left(C^{*}\right)$ has a corresponding enumeration 
$\lambda_{1}^{-1}, \lambda_{2}^{-1}, \ldots$. Let $N_{i}=\operatorname{ker}\left(C^{*}-\lambda_{i}^{-1}\right)=\operatorname{ker} U\left(B^{-1}-\lambda_{i}^{-1}\right) U^{*}$. There is a decomposition $Y=\left[Y_{i j}\right]$ of $Y: \bigoplus_{i} N_{i} \rightarrow \bigoplus_{i} M_{i}$ such that

$$
\left[\begin{array}{ccc}
\lambda_{1} & & 0 \\
& \lambda_{2} & \\
0 & & \ddots
\end{array}\right]\left[\begin{array}{ccc}
Y_{11} & Y_{12} & \ldots \\
Y_{21} & Y_{22} & \ldots \\
\vdots & & \ddots
\end{array}\right]\left[\begin{array}{ccc}
\lambda_{1}^{-1} & & 0 \\
& \lambda_{2}^{-1} & \\
0 & & \ddots
\end{array}\right]=\left[\begin{array}{ccc}
Y_{11} & Y_{12} & \ldots \\
Y_{21} & Y_{22} & \cdots \\
\vdots & & \ddots
\end{array}\right] \text {. }
$$

Since the $\lambda_{i}$ 's are distinct, this implies that $Y_{i j}=0$ for all $i \neq j$. Consider now $R\left(A, A^{*}\right)(X)+S$. Since

$$
\left\|R\left(A, A^{*}\right)(X)+S\right\|=\left\|R\left(A^{\circ}, A^{\circ *}\right)\left(X^{\circ}\right)+S^{\circ}\right\|
$$

we have, upon letting $X^{\circ}: \operatorname{ker}^{\perp} S^{\circ} \oplus \operatorname{ker} S^{\circ} \rightarrow \overline{\operatorname{ran} S^{\circ}} \oplus\left(\overline{\operatorname{ran} S^{\circ}}\right)^{\perp}$ have the representation $X^{\circ}=\left[X_{i j}\right]_{i, j=1}^{2}$, that

$$
\left\|R\left(A, A^{*}\right)(X)+S\right\|=\left\|\left[\begin{array}{cc}
R\left(B, C^{*}\right)\left(X_{11}\right)+Y & * \\
* & R\left(B_{\circ}, C_{\circ}^{*}\right)\left(X_{22}\right)
\end{array}\right]\right\| .
$$

Here $R\left(B, C^{*}\right)\left(X_{11}\right)$ is a matrix whose diagonal entries are 0 and the matrix of $Y$ has nothing but diagonal entries. Recall that the norm of an operator matrix is always greater than or equal to the norm of the operator matrix consisting of its diagonal entries only [4, p. 82]; applying this twice, we have from the norm equality above that

$$
\begin{aligned}
\left\|R\left(A, A^{*}\right)(X)+S\right\| & \geq \max \left(\left\|R\left(B, C^{*}\right)\left(X_{11}\right)+Y\right\|,\left\|R\left(B_{\circ}, C_{\circ}^{*}\right)\left(X_{22}\right)\right\|\right) \\
& \geq \|\left(R\left(B, C^{*}\right)\left(X_{11}\right)+Y\|\geq\| Y\|=\| S^{\circ}\|=\| S \| .\right.
\end{aligned}
$$

This completes the proof.

Corollary 1. If $A, B$ are normal operators such that $R(A, B)(S)=0$ for some $S \in B(H)$, then

$$
\|R(A, B)(X)+S\| \geq\|S\|
$$

for all $X \in B(H)$.

Proof. Consider

$$
\left\|\left[\begin{array}{cc}
A & 0 \\
0 & B^{*}
\end{array}\right]\left[\begin{array}{cc}
0 & X \\
0 & 0
\end{array}\right]\left[\begin{array}{cc}
A^{*} & 0 \\
0 & B
\end{array}\right]-\left[\begin{array}{cc}
0 & X \\
0 & 0
\end{array}\right]+\left[\begin{array}{cc}
0 & S \\
0 & 0
\end{array}\right]\right\|
$$

on $\widehat{H}=H \oplus H$.

Choosing $B=A$ in Corollary 1, inequality (4) reduces to inequality (2) (albeit with an improved estimate). As shown in [2], a proof of Corollary 1 may be obtained from Anderson's inequality. We show next the equivalence of Corollary 1 and Anderson's theorem (by showing that Corollary 1 implies Anderson's theorem).

Corollary 2. If $A, B$ are normal operators such that $C(A, B)(S)=0$ for some $S \in B(H)$, then

$$
\|C(A, B)(X)+S\| \geq\|S\|
$$

for all $X \in B(H)$. 
Proof. It is enough to prove that if $A$ is a normal operator such that $C\left(A, A^{*}\right)(S)=$ 0 for some $S \in B(H)$, then $\left\|C\left(A, A^{*}\right)(X)+S\right\| \geq\|S\|$ for all $X \in B(H)$ (argue as in the proof of Corollary 1$)$. The Berberian extension theorem implies that $C\left(A^{\circ}, A^{\circ *}\right)\left(S^{\circ}\right)=0$, where $A^{\circ}$ is normal with $\sigma\left(A^{\circ}\right)=\sigma_{p}\left(A^{\circ}\right)$. If $0 \in \sigma\left(A^{\circ}\right)$, then there is a reducing subspace $M$ (of $H^{\circ}$ ) such that $A^{\circ} \mid M=0$ and $A_{2}=A^{\circ} \mid M^{\perp}$ is an invertible normal operator. Let $S^{\circ}=\left[S_{i j}\right]_{i, j=1}^{2}$ on $H^{\circ}=M \oplus M^{\perp}$. Since $C\left(A^{\circ}, A^{\circ *}\right)\left(S^{\circ}\right)=0, S_{i j}=0$ for all $i \neq j$ and $C\left(A_{2}, A_{2}^{*}\right)\left(S_{22}\right)=0$. Letting $X^{\circ}$ have the representation $X^{\circ}=\left[X_{i j}\right]_{i, j=1}^{2}$ (on $H^{\circ}=M \oplus M^{\perp}$ ) we now have that

$$
\begin{aligned}
\left\|C\left(A, A^{*}\right)(X)+S\right\|= & \left\|C\left(A^{\circ}, A^{\circ *}\right)\left(X^{\circ}\right)+S^{\circ}\right\| \\
= & \left\|\left[\begin{array}{cc}
S_{11} & * \\
* & C\left(A_{2}, A_{2}^{*}\right)\left(X_{22}\right)+S_{22}
\end{array}\right]\right\| \\
\geq & \left\|S_{11} \oplus\left\{C\left(A_{2}, A_{2}^{*}\right)\left(X_{22}\right)+S_{22}\right\}\right\| \\
= & \left\|S_{11} \oplus\left\{R\left(A_{2}, A_{2}^{*-1}\right)\left(X_{22} A_{2}^{*}-S_{22}\right)+S_{22}\right\}\right\| \\
& \quad\left(\text { since } C\left(A_{2}, A_{2}^{*}\right)\left(S_{22}\right)=0 \Rightarrow R\left(A_{2}, A_{2}^{*-1}\right)\left(S_{22}\right)=0\right) \\
\geq & \max \left\{\left\|S_{11}\right\|,\left\|S_{22}\right\|\right\} \quad(\text { by Corollary } 1) \\
= & \left\|S^{\circ}\right\|=\|S\| . \quad \square
\end{aligned}
$$

Corollary 1 extends to non-normal pairs $\left(A, B^{*}\right)$ which satisfy a Putnam-Fuglede theorem type commutativity property. Thus let us call $\left(A, B^{*}\right)$ a PF-pair if $R(A, B)(X)=0, X \in B(H)$, implies $R\left(A^{*}, B^{*}\right)(X)=0$. Hyponormal (even $M$-hyponormal) operators $A, B^{*}$ define a PF-pair $\left(A, B^{*}\right)[3]$.

Corollary 3. For every PF-pair $\left(A, B^{*}\right)$ such that $R(A, B)(S)=0$ for some $S \in$ $B(H)$,

$$
\|R(A, B)(X)+S\| \geq\|S\|
$$

for all $X \in B(H)$.

Proof. Using the Berberian extension theorem we have that $R\left(A^{\circ}, B^{\circ}\right)\left(S^{\circ}\right)=0=$ $R\left(A^{\circ *}, B^{\circ *}\right)\left(S^{\circ}\right)$. Thus $\overline{\operatorname{ran} S^{\circ}}$ reduces $A^{\circ}$ and $\operatorname{ker}^{\perp} S^{\circ}$ reduces $B^{\circ}$. Let $A_{1}=$ $A^{\circ}\left|\overline{\operatorname{ran} S^{\circ}}, B_{1}=B^{\circ}\right| \operatorname{ker}^{\perp} S^{\circ}$ and let $S_{1}: \operatorname{ker}^{\perp} S^{\circ} \rightarrow \overline{\operatorname{ran} S^{\circ}}$ be the quasi-affinity defined by setting $S_{1} x=S^{\circ} x$ for each $x \in \operatorname{ker}^{\perp} S^{\circ}$. Then $R\left(A_{1}, B_{1}\right)\left(S_{1}\right)=0=$ $R\left(A_{1}^{*}, B_{1}^{*}\right)\left(S_{1}\right)$, and it follows that $0 \notin \sigma\left(A_{1}\right)$ and $0 \notin \sigma\left(B_{1}\right)$. Since the PF-pair property applied to $R\left(A^{\circ}, B^{\circ}\right)\left(A^{\circ} S^{\circ}\right)=0$ implies $A_{1}^{*} A_{1} S_{1}=A_{1} S_{1} B_{1}^{*-1}=A_{1} A_{1}^{*} S_{1}$, $A_{1}$ is normal. Similarly, $B_{1}$ is normal.

Let $X^{\circ}=\operatorname{ker}^{\perp} S^{\circ} \oplus \operatorname{ker} S^{\circ} \rightarrow \overline{\operatorname{ran} S^{\circ}} \oplus\left(\overline{\operatorname{ran} S^{\circ}}\right)^{\perp}$ have the representation $X^{\circ}=$ $\left[X_{i j}\right]_{i, j=1}^{2}$. Then

$$
\begin{aligned}
\|R(A, B)(X)+S\| & =\left\|R\left(A^{\circ}, B^{\circ}\right)\left(X^{\circ}\right)+S^{\circ}\right\| \\
& =\left\|\left[\begin{array}{cr}
R\left(A_{1}, B_{1}\right)\left(X_{11}\right)+S_{1} & * \\
* & *
\end{array}\right]\right\| \\
& \geq\left\|R\left(A_{1}, B_{1}\right)\left(X_{11}\right)+S_{1}\right\| \\
& \geq\left\|S_{1}\right\|=\left\|S^{\circ}\right\|=\|S\| . \quad \square
\end{aligned}
$$

Given a (not necessarily normal) contraction $A$, if $R\left(A, A^{*}\right)(S)=0=R\left(A^{*}, A\right)(S)$ for some $S \in B(H)$, then $\overline{\operatorname{ran} S}$ reduces $A, A$ commutes with $S$ and $A \mid \overline{\operatorname{ran} S}$ is unitary. This is seen as follows. Let $A$ have the polar decomposition $A=U P$. 
Let $x \in H$, and let $x_{n}=S P^{2 n} x(n=0,1,2, \ldots)$. Since $A S A^{*}=A^{*} S A$ implies $P^{2} S P^{2}=S, P^{2} x_{n+1}=x_{n}$ and the sequence $\left\{\left\|x_{n}\right\|\right\}$ is monotonic increasing. Also, since

$$
\left\|x_{n+1}\right\|^{2}=\left(x_{n+2}, P^{2} x_{n+1}\right)=\left(x_{n+2}, x_{n}\right) \leq\left(\frac{\left\|x_{n}\right\|+\left\|x_{n+2}\right\|}{2}\right)^{2},
$$

$\left\{\left\|x_{n}\right\|\right\}$ is a convex sequence. Hence $\left\{\left\|x_{n}\right\|\right\}$ is a constant sequence. In particular, $\|S x\|=\left\|S P^{2} x\right\|$. Since this implies

$$
\left\|\left(P^{2} S-S P^{2}\right) x\right\|^{2}=\left\|P^{2} S x\right\|^{2}+\left\|S P^{2} x\right\|^{2}-2 \operatorname{Re}\left(P^{2} S P^{2} x, S x\right) \leq 0,
$$

$P^{2} S=S P^{2}$, i.e., $P$ commutes with $S$. The commutativity of $S$ and $P$ implies that $P^{4 n} S=S$ for all $n=0,1,2, \ldots$ Hence $P S=S(=S P)$. The equation $R\left(A, A^{*}\right)(S)=0$ now implies that $S=U P S P U^{*}=U S U^{*}$ and $S^{*}=U P S^{*} P U^{*}=$ $U S^{*} U^{*}$. Thus $U^{*} S=S U^{*}$ and $U S=S U$, and so both $A$ and $A^{*}$ commute with $S$. This, since $A A^{*} S=A S A^{*}=S=A^{*} S A=A^{*} A S$, implies that $\overline{\operatorname{ran} S}$ reduces $A$ and $A \mid \overline{\operatorname{ran} S}$ is unitary.

The following corollary is now immediate from Corollary 3.

Corollary 4. If $A$ is a contraction such that $R\left(A, A^{*}\right)(S)=0=R\left(A^{*}, A\right)(S)$ for some $S \in B(H)$, then

$$
\left\|R\left(A, A^{*}\right)(X)+S\right\| \geq\|S\|
$$

for all $X \in B(H)$.

Unitarily invariant norms. Each unitarily invariant norm $|\| \cdot|||$ satisfies the property that $\||U A|\|=|\|A V \mid\|$ for all unitaries $U$ and $V$ (provided that $\||A|\|<\infty$ ), and is defined on a natural subclass $J_{\||| \cdot||}$ of $B(H)$, called the norm ideal associated with $|\|\cdot|\||$. Whereas the (unitarily invariant) usual operator norm $\| \cdot \|$ is defined on all of $B(H)$, other unitarily invariant norms are defined on norm ideals contained in the ideal $K(H)$ of compact operators in $B(H)$. (For further information on unitarily invariant norms see [4].) Kittaneh [5] has shown that inequality (1) extends to unitarily invariant norms: The following theorem proves the $R\left(A, A^{*}\right)$ analogue of inequality (1) for unitarily invariant norms.

Theorem 2. Let $A$ be a normal operator such that $R\left(A, A^{*}\right)(S)=0$ for some $S \in B(H)$, and let $X \in B(H)$. If $R\left(A, A^{*}\right)(X)+S \in J_{\||| \cdot \mid\|}$, then $\left(S \in J_{\||\cdot|\| \mid}\right.$ and $)$

$$
\left\|R\left(A, A^{*}\right)(X)+S|\|\geq\|| S\right\| \mid .
$$

Proof. Clearly, $\overline{\operatorname{ran} S}$ reduces $A$ and $\operatorname{ker}^{\perp} S$ reduces $A^{*}$. Let $A=B \oplus B_{\circ}$ on $H=\overline{\operatorname{ran} S}+(\overline{\operatorname{ran} S})^{\perp}$ and let $A^{*}=C^{*} \oplus C_{\circ}^{*}$ on $H=\operatorname{ker}^{\perp} S \oplus \operatorname{ker} S$. Defining the quasi-affinity $Y: \operatorname{ker}^{\perp} S \rightarrow \overline{\operatorname{ran} S}$ by setting $Y x=S x$ for each $x \in \operatorname{ker}^{\perp} S$, it follows that $R\left(B, C^{*}\right)(Y)=0$ (where $B$ and $C^{*-1}$ are unitarily equivalent normal operators - see the proof of Theorem 1). Let $H_{1}=\bigvee_{\lambda \in \mathcal{C}} \operatorname{ker}(B-\lambda)$ and let $H_{2}=$ $\overline{\operatorname{ran} S} \ominus H_{1}$; then $H_{1}$ reduces $B, B_{1}=B \mid H_{1}$ has pure point spectrum and $B_{2}=$ $B \mid H_{2}$ has empty point spectrum. Similarly, let $H_{1}^{\prime}=\bigvee_{\lambda \in \mathcal{C}} \operatorname{ker}\left(C^{*}-\lambda\right)$ and let $H_{2}^{\prime}=\operatorname{ker}^{\perp} S \ominus H_{1}^{\prime}$; then $H_{1}^{\prime}$ reduces $C^{*}, C_{1}^{*}=C^{*} \mid H_{1}^{\prime}$ has pure point spectrum and $C_{2}^{*}=C^{*} \mid H_{2}^{\prime}$ has empty point spectrum. Letting $Y: H_{1}^{\prime} \oplus H_{2}^{\prime} \rightarrow H_{1} \oplus H_{2}$ have the representation $Y=\left[Y_{i j}\right]_{i, j=1}^{2}$, it then follows that $Y_{i j}=0$ for all $i \neq j$. (For example: $B_{1} Y_{12} C_{2}^{*}=Y_{12} \Rightarrow B_{1} Y_{12}=Y_{12} C_{2}^{*-1}$ and $B_{1}^{\star} Y_{12}=Y_{12} C_{2}^{-1}$. Thus $B_{1} \mid \overline{\operatorname{ran} Y_{12}}$ and $C_{2}^{*-1} \mid \operatorname{ker}^{\perp} Y_{12}$ are unitarily equivalent. Since $\sigma_{p}\left(C_{2}^{*}\right)=\varnothing$ and $\sigma\left(B_{1}\right)=\sigma_{p}\left(B_{1}\right), Y_{12}=0$.) We prove next that $Y_{22}=0$. 
From above, $B_{2} Y_{22} C_{2}^{*}=Y_{22}$; hence $B_{2} Y_{22}-Y_{22} C_{2}^{*-1}=0=B_{2}^{*} Y_{22}-Y_{22} C_{2}^{-1}$,

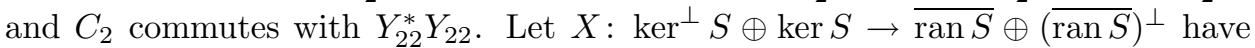
the representation $X=\left[\begin{array}{c}Z * \\ * *\end{array}\right]$; then $R\left(A, A^{*}\right)(X)+S \in J_{\||\cdot|\| \mid}$ implies (in particular) that $R\left(B, C^{*}\right)(Z)+Y \in J_{\||\cdot|\| \mid} \subset K(H)$. Applying Corollary 1 to the Calkin algebra $B(H) / K(H)$ we conclude that $Y$, and hence $Y_{22}$, is compact. Since $\sigma_{p}\left(C_{2}\right)=\varnothing$ and $C_{2}$ commutes with $Y_{22}^{\star} Y_{22}, Y_{22}^{\star} Y_{22}=0$. Hence $Y_{22}=0$.

Let $Z: \operatorname{ker}^{\perp} S \rightarrow \overline{\operatorname{ran} S}$ have the representation $Z=\left[Z_{i j}\right]_{i, j=1}^{2}$; then

$$
\begin{aligned}
& \left\|\left|R\left(A, A^{*}\right)(X)+S\right|\right\|=\left\|\begin{array}{ccc}
R\left(B_{1}, C_{1}^{*}\right)\left(Z_{11}\right)+Y_{11} & * & * \\
* & * & * \\
* & * & *
\end{array} \mid\right\| \\
& \geq\|\mid\| R\left(B_{1}, C_{1}^{*}\right)\left(Z_{11}\right)+Y_{11}\|\| .
\end{aligned}
$$

As seen above, $R\left(B_{1}, C_{1}^{*}\right)\left(Y_{11}\right)=0$. Hence $B_{1}$ and $C_{1}^{*-1}$ are unitarily equivalent normal operators; in particular, $\sigma\left(B_{1}\right)=\sigma_{p}\left(B_{1}\right)=\sigma_{p}\left(C_{1}^{*-1}\right)=\sigma\left(C_{1}^{*-1}\right)$. Arguing as in the proof of Theorem 1, we have

$$
\text { || } R\left(B_{1}, C_{1}^{*}\right)\left(Z_{11}\right)+Y_{11}|\|\geq\||\left|Y_{11}\right|\|=|\|Y\||=|\|S \mid\| .
$$

The argument above shows inequality $\left(2^{\prime \prime}\right)$ holds with equality in the case in which $\||\cdot|\|$ is the Hilbert-Schmidt norm. There is a PF-pair analogue of Theorem 2 for unitarily invariant norms, but we leave its formulation to the reader.

It is my pleasure to thank the referee for his suggestions, which have helped improve the presentation of the proof of Theorem 1.

\section{REFERENCES}

1. J. Anderson, On normal derivations, Proc. Amer. Math. Soc. 38 (1973), 135-140. MR 47:875

2. Du Hong-Ke, Another generalization of Anderson's theorem, Proc. Amer. Math. Soc. 123 (1995), 2709-2714. MR 95k:47032

3. Jin Chuan Hou, On the Putnam-Fuglede theorems for non-normal operators (Chinese), Acta Math. Sinica 28 (1985), 333-340. MR 87b:47022

4. I. C. Gohberg and M. G. Krein, Introduction to the Theory of Linear Non-selfadjoint Operators, Transl. Math. Monographs, Vol. 18, Amer. Math. Soc., Providence, RI, 1969. MR 39:7447

5. Fuad Kittaneh, Normal derivations in norm ideals, Proc. Amer. Math. Soc. 123 (1995), 17791785. MR 95g:47054

6. D. Xia, Spectral Theory of Hyponormal Operators, Birkhäuser Verlag (Basel), 1983. MR $\mathbf{8 7 \mathbf { j }}: 47036$

Department of Mathematics and Statistics, College of Science, Sultan Qaboos University, P.O. Box 36, Al-Khod 123, Oman

E-mail address: duggbp@squ.edu.om 